This is the peer reviewed version of the following article: FULL CITE, which has been published in final form at https://doi.org/10.1111/jocn.15089. This article may be used for non-commercial purposes in accordance With Wiley Terms and Conditions for self-archiving.

\title{
UNMET NURSING CARE NEEDS ON MEDICAL AND SURGICAL WARDS: A SCOPING REVIEW OF PATIENTS' PERSPECTIVES
}

\begin{abstract}
Aims and objectives

Review and synthesize research studies on surgical and medical inpatients' perceptions on unmet nursing care needs.
\end{abstract}

\section{Background}

Missed nursing care is a growing phenomenon that has been shown to adversely affect care outcomes - mainly in adult medical and surgical care settings. However, to date the aggregated and synthesized evidence of missed care comes from research that measures perceptions on missed care in surgical and medical settings from nurses, but not from the patients.

\section{Design}

Scoping review.

\section{Methods}

In September 2018, three databases were searched: MEDLINE/PubMed, CINAHL, and SCOPUS and papers were selected using the Preferred Reporting Items for Systematic Reviews and Meta-Analyses guidelines. Inclusion criteria: primary studies; published in peer-reviewed journals; in English or Italian; regarding routine care provided to adult inpatients. Quality appraisal and a thematic analysis were conducted. 


\section{Results}

Of the 1541 abstracts initially identified, 44 papers were included. Five themes emerged: 'communication', 'self-management, autonomy, and education', 'personal sphere', 'essential physical care', and 'emotional and psychological care'. The majority of the unmet needs were related to the 'personal sphere' and 'emotional and psychological care'. These unmet needs were not identified in previous literature on nurses' perspectives of missed care. Also physical care deficits like oral hygiene were identified.

\section{Conclusion}

It is important to take into account patients' perspectives. The themes focusing on patients' personal sphere, and emotional and psychological care, underline how patients need nurses to pay more attention to their cultural background, consider the person as a whole, and for nursing care to be holistic and respectful of patients' dignity.

\section{Relevance to Clinical Practice}

This study intends to raise awareness among nurses and policy makers about the importance of addressing missed nursing care and unmet patients' needs in adult medical or surgical inpatient settings to ensure high quality care and patient satisfaction.

Key words: Scoping review, patient needs, satisfaction, nursing care, missed nursing care, quality of care, fundamental of care 


\section{SUMMARY BOX}

\section{What does this paper contribute to the wider global clinical community?}

- It is very important to take into account the perspectives patients have of the health services they receive to improve the quality of care.

- Often patients describe needs that are not perceived as such by nurses and remain unmet.

- Being aware of patients' perspectives, health professionals will be able to respond more effectively to patients' needs.

\section{INTRODUCTION}

In the last ten years, there has been a growth of research that examines missed nursing care. This work has been prompted by numerous, high profile cases where there has been a lack of fundamental, essential nursing care - in some cases resulting in national scandal as in the MidStaffordshire case in the UK - where patients failed to receive the most basic levels of care (Hayter, 2013). In response to this issue, researchers have focused significant attention on investigating the impact of deficiencies in nursing care on patient outcomes. The majority of this work has been done in inpatient adult medical and surgical areas - notably the highly influential RN4CAST studies designed to investigate the quality and safety of nursing care performed in adult medical and surgical settings (Sermeus et al., 2011; Aiken et al., 2014). A key issue that emerged from this study is the phenomena of 'missed nursing care' and the detrimental effect it can have on patient care outcomes, like falls or infections (Griffiths et al., 2018b).

The amount of missed nursing care, identified by several studies, is often high; across studies that measured missed nursing care with a nurse-completed survey tool in the RN4CAST Consortium project, the estimated frequency of care omissions ranged from $75 \%$ in England 
(Ball et al., 2014), to 93\% in Germany (Zander et al., 2014), to an overall of 88\% in 12 European countries (Griffiths, et al., 2014), and to $81 \%$ in South Korea (Cho et al., 2016). The nature and type of missed nursing care across the literature was quite consistent with 'comfort and talk with patients' missed by $53 \%$ of nurses from twelve European countries (Belgium, England, Finland, Germany, Greece, Ireland, The Netherlands, Norway, Poland, Spain, Sweden and Switzerland) (Ausserhofer et al., 2014), by 44\% of Italian nurses (Sasso et al., 2017), and by $65 \%$ of UK nurses (Aiken et al., 2018). Care related to "educating patients and families" was missed by 41\% (Ausserhofer et al., 2014), 45\% (Sasso et al., 2017), and 52\% (Aiken et al., 2018), respectively. The relationship between missed nursing care and mortality rates has also been extensively studied, and Ball et al. (2018) confirmed that a $10 \%$ increase in the frequency of missed nursing care is associated with a $16 \%$ increase in the odds of mortality in medical and surgical care environments. Several other studies in adult medical and surgical settings have also identified factors such as skill mix, work environment, nurse staffing and intention-to-leave that can contribute to missed care (Papastavrou, Andreou, \& Efstathiou, 2014; Kalisch \& Xie, 2014; Jones, Hamilton, \& Murry, 2015; Griffiths et al., 2018b; Suhonen et al., 2018; Sasso et al., 2019). The missed care phenomenon has also led to calls for nursing to re-focus on developing a better understanding of how failing to address the fundamental aspects of nursing care effects patient experience (Kitson, 2016; Feo, Kitson \& Conroy, 2018).

However, to date, the aggregated and synthesized evidence of missed care comes from research that only measures nurses' perceptions on missed care in surgical and medical settings - not from the patient perspective (Jones et al., 2015; Griffiths et al. 2018b; Suhonen et al., 2018). Although there is a body of literature on how patients perceive unmet care needs, there are no reviews that amalgamate or synthesis this evidence to identify the key issues on unmet care from 
the patient's perspective. Therefore, there is a need to complement the cumulative knowledge base regarding missed nursing care in medical and surgical settings from health professional's perspectives with that from the patient's perspective (Kalisch, McLaughlin \& Dabney, 2012; Kalisch, et al, 2014; Dabney \& Kalisch, 2015). Furthermore, there are no papers that compare patient experiences of unmet care needs with the literature on missed care based on professionals' perspectives. Ensuring that the patient's voice is included in the missed care/unmet care needs debate is important, thus, there is a need to summarise, amalgamate and synthesise the empirical work on patient's views on missed care to contribute to our understanding of this phenomenon. This paper is designed to address this gap by conducting a scoping review to answer the following question: "which nursing care needs do medical and surgical inpatients perceive as unmet?"

\subsection{AIMS}

To review and synthesize research evidence on adult medical and surgical inpatients' perceptions of unmet nursing care needs and compare these with the literature on nurses' perspectives about missed care.

\section{METHODS}

\subsection{Design}

A scoping review approach was used, according to the methodological framework proposed by Arksey and O’Malley (Arksey \& O’Malley, 2005), which includes five steps to identify, select, 
and revise the literature: 1) research question identification; 2) identification of relevant studies; 3) study selection; 4) data collection; 5) data synthesis and summary of the findings. Papers were selected according to the Preferred Reporting Items for Systematic Reviews and Meta-Analyses guidelines (see Supplementary File 1).

\subsection{Search strategy}

The search strategy included keywords base on the Population, Exposure and Outcomes (PEO) matrix (Table 1). In September 2018, three databases were searched: MEDLINE/ PubMed, CINAHL, and SCOPUS (Table 2). The initial database search identified 1541 abstracts, and none were identified elsewhere (Figure 1). After removing the duplicates, another 1256 abstracts were removed due to exclusion criteria. The remaining papers were screened for full-text eligibility ( $n=172$ ). Another 128 articles were excluded following full-text review because they did not meet the inclusion criteria. In the end, 44 papers were included in this study for data extraction and analysis.

At least two authors reviewed all the papers throughout the above-mentioned steps, independently. At the end of each step, the screening results were compared between the research members until agreement was reached.

INSERT TABLE 1 AND TABLE 2 ABOUT HERE

\subsection{Inclusion criteria}

Inclusion criteria: primary studies, papers published in peer reviewed journals, in English or Italian, papers related to routine care provided in adult medical or surgical inpatient settings. 
Exclusion criteria: papers on nursing education or nursing students, papers not dealing with nursing care, papers that do not consider the patients' point of views, and those published before 2009, because this was when the RN4CAST studies started (Bruyneel et al., 2009; Sermeus et al., 2011), and the concept of missed nursing care was described for the first time (Kalisch, Landstrom, \& Hinshaw, 2009).

\subsection{Quality appraisal}

During data extraction, papers underwent a quality appraisal process. Although scoping reviews do not normally require this level of scrutiny, we decided to conduct one to give some rigour and quality context to our findings. For the qualitative studies, we used the CASP checklist for Qualitative Studies (CASP, 2018), whereas for the quantitative papers we used Kelley Survey reporting (Kelley, Clark, Brown, \& Sitzia, 2003). No mixed-methods studies were found. Table 3 outlines the quality questions that were asked of each study.

INSERT HERE TABLE 3 ABOUT HERE

\subsection{Data abstraction \& synthesis}

Arksey and O'Malley refer to this stage as an "analytical descriptive method", which involves informing the synthesis process according to a specific format (Arksey \& O'Malley, 2005). The team of reviewers jointly develops a data collection table to determine which variables to extract to answer the research question, and to conduct a descriptive analysis. The information provided in Table 4 includes: references and countries, study design and aim, participants/setting and data collection, met/unmet needs and general findings. Subsequently, a thematic analysis was 
performed, by two authors to identify patterns, trends, and variation in patient experience about met or unmet needs. Our starting point was the result sections of the included papers. We analysed what needs were investigated, how they were described and measured. Then we coded needs as met or unmet and continued until we defined our main themes. This analysis allowed us to describe the voice of patients about how nurses take charge and satisfy their care needs. Then, this initial analysis was compared with that of the other author and then with the group who then met to agree on the final thematic analysis of the findings.

\section{RESULTS}

\subsection{Study characteristics}

Of the 44 papers included in the review, 16 were qualitative studies (most of which were descriptive), and 28 were quantitative studies (which mostly had a cross-sectional observational design). The included studies were conducted in different geographical areas: 18 studies were conducted in various European countries; 8 studies were conducted in the United States, 8 in the Middle East, 5 in East Asia, and one in Canada. A summary of the studies and the types of missed care are shown in Table 4.

\section{INSERT TABLE 4 ABOUT HERE}

After conducting quality appraisal, we calculated the number of sections of each study that received a positive evaluation. In general, the quality of quantitative studies was judged to be higher than that of qualitative studies: the average number of positive responses was $8 / 9$ for the quantitative studies, and 7/9 for the qualitative studies (See Tables 5 and 6).

INSERT TABLES 5 AND 6 ABOUT HERE 


\subsection{Thematic Analysis - Identifying the patients' perceptions about met and unmet needs by nursing care}

From the thematic analysis, five themes were identified representing the patients' perspectives of their own unmet nursing care needs (see Table 7).

\section{INSERT TABLE 7 ABOUT HERE}

\subsubsection{Communication}

Communication was a key aspect of unmet needs identified by many patients across the studies. Communication is identified as the first step in establishing a relationship between patient and nurse, therefore, establishing a communicative relationship is fundamental for ensuring quality care (Dabney \& Kalisch, 2015; Dorigan \& De Brito Guirardello 2010; Ebrahimi et al. 2012; Goh et al. 2016; Mbuzi et al. 2017; Oflaz \& Vural 2010; Papastavrou et al. 2012; Suhonen et al. 2009). They often felt communication could be better between themselves and nurses in relation to a range of care related issues. For example, patients wanted to have more information about their care plans and the procedures they were going to undergo. They also wanted more discussion with nurses about their medication and also the organization and internal rules of the ward (Abdel, Oweis, \& Hasna, 2012; Alasad, Tabar, \& Aburuz, 2015; Alhusban, \& Abualrub, 2009; Bachnick et al., 2018; Bishop \& Macdonald, 2017; Cano-Plans et al., 2018; Crispin, Bugge, \& Stoddart, 2017; Dorigan, \& De Brito Guirardello, 2010; Fuji, Abbott, \& Norris, 2013; Gellerstedt, Medin, \& Karlsson, 2014; Goh et al., 2016; Johansson Stark et al., 2014; Kalisch et al., 2012; Laird et al., 2014; Land \& Suhonen, 2009; Lin, Tsai, \& Chen, 2011; Mahjoub \& Rutledge, 2011; Malmgren Törnvall, \& Jansson, 2014; Mbuzi, Fulbrook, \& Jessup, 2017; Montin et al., 2010; Mukasa 
Glass, \& Mnatzaganian, 2015; Oetker-Black, \& Petrochuk, 2012; Oflaz \& Vural, 2010; Palese $e t$ al., 2011; Portillo \& Cowley, 2011; Ringdal et al., 2017; Sayin \& Aksoy, 2012; Shoqirat, 2014; Suhonen et al., 2012; Van Bokhorst-De Van Der Schueren et al., 2012; Yiu et al., 2011; Zhang et al., 2009). Furthermore, merely 'passing information' was not sufficient for many patients. They felt the use of technical terms and jargon affected their ability to fully understand what they were being told (Bachnick et al., 2018; Crispin et al., 2017; Dorigan, \& De Brito Guirardello, 2010). They also wanted nurses to spend more time with them discussing their care and to be listened (Alasad et al., 2015; Alhusban, \& Abualrub, 2009; Bishop \& Macdonald, 2017; Dorigan, \& De Brito Guirardello, 2010; Kalisch et al., 2012; Koskenniemi et al., 2018; Laird et al., 2014; Malmgren et al., 2014; Oflaz \& Vural, 2010; Ringdal et al., 2017). Patients often felt that their need to develop a relationship, a 'connection', with the healthcare team was not possible due to a lack of in-depth communication and relationship building by the nursing team (Majhoub et al., 2011, Bishop \& Macdonald, 2017,).

\subsubsection{Self-Management, autonomy and education}

During hospitalization, patients wanted to manage themselves as autonomously as possible, to maintain control over their lives, be actively involved in the care process, in their care plan, and therefore in the decision-making process. However, these needs were often not adequately met with patients identifying a lack of autonomy and involvement in decision making across many of the studies (Bachnick et al., 2018; Bishop \& Macdonald, 2017; Bruus et al., 2012; Crispin et al., 2017; Goh et al., 2016; Laird et al., 2014; Land \& Suhonen, 2009; Lin et al., 2011; Mahjoub \& Rutledge, 2011; Malmgren et al., 2014; Palese et al., 2011; Rasmussen \& Delmar, 2018; Ringdal et al., 2017). Patients stated their need to return to a more autonomous state of being and to be 
more able to manage themselves and their needs (Alhusban, \& Abualrub 2009; Bozimowski 2012; Bruus et al. 2012; Goh et al. 2016; Kalisch et al. 2012; Montin et al. 2010; Mukasa et al. 2015; Palese et al. 2011; Portillo \& Cowley 2011; Shoqirat 2014; Suhonen, et al. 2012; Van Bokhorst-de van der Shueren et al. 2012; Yiu et al. 2011; Zhang et al. 2009). Another area that patients identified as an unmet need was the preparation and involvement in their discharge. Many studies reported that patients felt marginalised during plans for their return home (Fuji et al., 2013; Malmgren et al., 2014; Mbuzi et al., 2017; Oflaz \& Vural, 2010; Portillo \& Cowley, 2011; Shoqirat, 2014). This unmet need continued also after discharge, with some patients feeling they were left alone to manage their treatment and care. Patients across the study expressed feelings of anxiety about a return home and identified that this was an important unmet need (Bozimowski, 2012; Bruss et al., 2011; Fuji et al., 2013; Goh et al., 2016; Kalisch et al., 2012; Mbuzi et al., 2017; Montin et al., 2010; Mukasa et al., 2015; Palese et al., 2011; Portillo \& Cowley, 2011; Suhonen et al., 2009; Van Bokhorst-De Van Der Schueren et al., 2012; Yiu et al., 2011; Zhang et al., 2009).

\subsubsection{Fundamental physical care}

The studies revealed that patients also identified unmet care needs in what could be described as 'basic or essential' care. Issues like help with hygiene, eating and drinking and help mobilising were present in many of the studies (Abdel et al., 2012; Abu-El-Noor \& Abu-El-Noor, 2014; Alasad et al., 2015; Alhusban, \& Abualrub, 2009; Bachninck et al., 2018; Bruus et al., 2012; Dabney \& Kalisch, 2015; Ebrahimi et al.,2012; Gellerstedt et al., 2014; Kalisch et al., 2012; Koskenniemi et al., 2018; Land \& Suhonen, 2009; Lei et al., 2009; Lin et al., 2011; Malmgren et al., 2014; Mbuzi et al., 2017; Oetker-Black, \& Petrochuk, 2012; Oflaz \& Vural, 2010; Palese et al., 2011; Papastavrou et al., 2012; Radecki, Reynolds, \& Kara, 2018; Rasmussen \& Delmar, 2018; Ringdal et al., 2017; Shoqirat, 2014; Suhonen et al., 2009; Suhonen et al., 2012; Van 
Bokhorst-De Van Der Schueren et al., 2012). Other unmet needs were, help with oral care and help with dressing - an issue that was seen as important for self-esteem and autonomy (Bozimowski, 2012; Gellerstedt et al., 2014; Kalisch et al., 2012; Mahjoub \& Rutledge, 2011; Malmgren et al., 2014; Papastavrou et al., 2012; Shoqirat, 2014). Another sub-theme merged into "fundamental physical care" is pain management. Many studies have investigated patients' perceptions of the efficacy of pain management interventions, patient empowerment in the management of therapy and symptoms, and nurses' attention to this symptom. Despite being among the most met needs, it is still only partially satisfied (Bozimowski 2012; Gellerstedt, et al. 2014; Kalisch et al. 2012; Mahjoub \& Rutledge 2011; Malmgren et al. 2014; Papastavrou et al. 2012; Shoqirat 2014).

\subsubsection{Emotional and psychological care}

Throughout the studies patients identified how they want to be treated as a 'person', an 'individual', whose dignity should be respected despite hospitalization and the situations of reduced autonomy. This often does not result to be adequately addressed in hospital settings (Alasad et al., 2015; Crispin et al., 2017; Koskienniemi et al., 2017; Land \& Suhonen, 2009; Malmgren et al., 2014; Papastavrou et al., 2012; Shoqirat, 2014; Suhonen et al., 2009; Suhonen et al., 2012). This theme includes also the need for emotional and spiritual support, which, in some included papers, is described as not always adequately met (Abu-El-Noor \& Abu-El-Noor, 2014; Crispin et al., 2017; Goh et al., 2016; Koskienniemi et al., 2017; Mukasa et al., 2015; Palese et al., 2011; Papastavrou et al., 2012; Portillo \& Cowley, 2011). Receiving good care in the emotional and psychological sphere allows the patient to develop a sense of safety and confidence towards the nursing staff and the care environment. When this sense of protection is lacking, it generates a psycho-physical malaise that is then reflected in the patient's well-being 
(Crispin et al. 2017; Dorigan \& De Brito Guirardello 2010; Gellerstedt et al. 2014; Laird et al. 2014; Suhonen et al. 2009; Twibell et al. 2015).

\subsubsection{The personal sphere}

A cross-cutting theme of nursing care involved aspects related to what can be labelled 'personal sphere'. Respect for privacy and care that recognised the importance of privacy in terms of maintaining dignity was an area of missed care identified by patients. This was a particular issue in medical and surgical settings where men and women share the same bed bays, and patients may experience embarrassment when they are seen by health workers of the opposite gender (Ebrahimi et al., 2012; Mbuzi et al., 2017; Yiu et al., 2011). The privacy of information was also an unmet care need for many patients who felt that their confidentiality was not always respected during their conversations with nurses (Abdel et al., 2012; Alasad et al., 2015; Crispin et al., 2017; Ebrahimi et al., 2012; Gellerstedt et al., 2014; Goh et al., 2016; Lin et al., 2011; Rasmussen \& Delmar, 2018; Ringdal et al., 2017). Many patients felt that their need for a care environment that ensured the quietness and rest they needed was missed by nurses (Gellerstedt $e t$ al., 2014; Ringdal et al., 2017). Some patients also identified that not being able to have family members at their bedsides was also an unmet need - often as a result of ward rules on visiting hours (Alhusban, \& Abualrub, 2009; Bruss et al., 2011; Ebrahimi et al., 2012; Mbuzi et al., 2017; Sayin \& Aksoy, 2012). There was also some evidence that patients' culturally related care needs are often not met (Mbuzi et al., 2017; Yiu et al., 2011).

\section{DISCUSSION}

Nursing is fundamentally about meeting the full care needs of patients to ensure their psychophysical wellbeing (Feo et al., 2018). This scoping review highlights how many patients 
in medical and surgical inpatient settings identify numerous areas where their nursing care needs are not met. The results highlight how nurses run the risk of missing some aspects of care, which affect several care domains including the communicative, emotional and psychological, and relational spheres. The results of the review, and in particular the themes regarding the 'personal sphere' and 'emotional and psychological care', underline how patients feel the need for nurses to pay more attention to their cultural background, and to consider the person as a whole, moving towards the provision of holistic nursing and respecting the dignity of the patient. It is important for patients to feel that they are being cared for in their entirety as persons, and not seen as an item on the 'to do' list (Crispin et al., 2017), or merely as a clinical condition to be dealt with (Lin et al., 2011). All this also involves the patient's need to feeling safe and protected throughout their stay in hospital (Crispin et al., 2017; Dorigan, \& De Brito Guirardello, 2010; Gellerstedt et al., 2014; Laird et al., 2014; Suhonen et al., 2009; Twibell et al., 2015), although hospitalization on the contrary has often been described as a place that generates feelings of unsafety due to uncertainty and lack of knowledge about hospital dynamics (Gellerstedt et al., 2014). With regard to the 'personal sphere', the first aspect to take into consideration is the language, which constitutes a barrier between the expression of a need, and it being met, especially in immigrants and ethnic minorities (Mbuzi et al., 2017). Attention to culture is a need that can negatively affect the patient's experience during hospitalization (Ebrahimi et al., 2012; Mbuzi et al., 2017; Yiu et al., 2011). However, previous studies on the phenomenon of missed nursing care from the perspective of nurses do not reveal this as an issue. Although the definition of missed nursing care describes these as necessary activities for the patient (Kalisch et al., 2009), and the fundamental care framework takes into account these needs (Feo et al., 2018), none of the instruments found in the literature (Kalisch, \& Williams, 2009; Schubert et al., 2007; 
Squires et al., 2012) allow for the evaluation of care activities that enable culturally congruent care.

The other three themes that emerged from our review of patients' perspectives of missed care were mostly congruent with those identified by nurses in other studies. 'Communication' appears to be an aspect of nursing that can be improved: quantitative studies about missed nursing care phenomenon have found that nurses are conscious about the omission of this important aspect of nursing care (Ausserhofer et al., 2014; Ball et al., 2014; Lake, Germack, \& Viscardi, 2016; Sasso et al., 2017). Patients interpret the need of communication not only as a need for a closer interaction and the building a relationship of trust with nurses, but also in terms of a greater participation in decisions that concern their care (Bruus et al., 2012; Crispin et al., 2017; Goh et al., 2016; Laird et al., 2014; Mahjoub \& Rutledge, 2011; Palese et al., 2011; Rasmussen \& Delmar, 2014; Ringdal et al., 2017). It is often noted that nurses attribute unmet care needs related to communication to a lack of time (Ringdal et al., 2017) however, from a patient perspective simply by spending time getting to know and talking with patients, professionals can earn the trust of their patients and are thereby more likely to involve them as partners in their care (Lin et al., 2011). A review (2013) on nursing communication behaviours in the acute care setting has previously highlighted how nurses' habits and attitudes might affect patient safety. During handover, fundamental parts of care, such as emotional or social support were often excluded. Moreover, the exclusion of the patient from the communication did not allow active participation of the patient in their care process of care - with the use of jargon and medical terms compounding this exclusion (Kitson, Muntlin Athlin, Elliott \& Cant, 2013). Communication is therefore urgent, and this is demonstrated by the fact that all the actors involved in the care process are aware of this. It is necessary to look for solutions that allow, on 
the one hand, making handover more effective and safer; on the other hand, to find solutions that allow the patient to feel listened to, involved and be an active part of the care process.

Hospital settings can be very disempowering and the literature included in this review reinforces that this is a common feeling amongst patients and that there are unmet nursing care needs that would have helped address these anxieties (Alhusban, \& Abualrub, 2009; Bruss et al., 2011; Portillo \& Cowley, 2011; Shoqirat, 2014; Suhonen et al., 2012). For example, the need to ensure patients feel as autonomous as possible was a care need that was often missed and was linked to patient's desires to 'being able to manage one's own life' (Yiu et al., 2011; Shoqirat, 2014). Moreover, the need for autonomy is also linked to having the ability to manage situations that will arise after they leave the safety of the hospital environment and return home (Portillo \& Cowley, 2011). This also links to the area of discharge planning and communication identified in several of the included studies as being a substantial unmet need.

Through this review we found that several of the unmet care needs identified within the patient perspective literature mapped closely with those identified by nurses, which is the source of some comfort for practitioners working in medical and surgical settings. Despite this, there were also other needs described by patients as unmet that did not intersect with the ones identified by nurses. On the other hand, there were some aspects of missed care identified by the nurses, but not by the patients. However, these were primarily about documentation, such as updating nursing care plans and completion of nursing charts (Ausserhofer et al., 2014; Sasso et al., 2017; Aiken et al., 2018) and it is therefore understandable that patients would not highlight this as they would not be aware of such missed care. Looking at the literature on both nurse and patient perspectives of care, some aspects are identified by both groups, whereas others sit outside the mutually reported areas (see Figure 2) 
This reinforces the need for a joint agenda to be developed in addressing these gaps in nursing care recognising that nursing is a partnership that relies on both parties understanding each other and being able to communicate effectively to ensure that both care and care needs are holistic and complete (Kitson \& Muntlin Athlin, 2013). The results of this review underline the importance of taking the patient's point of view into account throughout the health care delivery process and, although research into missed care from the professional perspective is valuable, we must also ensure we check that against the experiences of patients too. Furthermore, this reflection should also involve the leaders and stakeholders of the profession, at all levels. When basic needs such as hygiene, nutrition, mobilization, communication are not recognized, the patient feels humiliated, ignored; missed care creeps into the patient-nurse relationship and undermines confidence, esteem, and the sense of security. Because of the complex interaction of basic nursing care, every time nurses interact with a patient, they perform a "complex intervention" within a complex system such as healthcare. Often this complexity remains invisible to the eyes of the nurses themselves and consequently remains invisible even for all the others, due to the lack of systematic traceability of nursing activities. The traceability of the provision of nursing interventions could allow an objective quantification of the economic resources necessary to guarantee quality care (Sasso et al., 2017). Models that take into account the complexity of patients' needs, such as the Fundamentals of Care Framework, could help to get assistance more effectively, also intervening on many activities (Needleman, 2016), which, like this revision highlighted, are omitted. Many of the recent health care scandals, particularly in the United Kingdom, have been related to the neglect of fundamental patient care (Hayter 2013). Our review highlights that patients often report unmet needs and highlights how nursing needs to constantly strive to develop models of working that reduce this type of care deficit. 


\subsection{Limitations}

Although this scoping review was conducted in line with the methodology proposed by Arksey and O’Malley (Arksey \& O’Malley, 2005), it has some limitations. First of all, we searched only mainstream research databases and did include other data sources (e.g. reference lists or the grey literature). Moreover, a consolidated practice in scoping reviews is the involvement of the stakeholders to obtain an in-depth discussion of the results, which can lead to the integration of further elements of reflection. However, this was not done due to lack of time and resources. Finally, although our review was designed to complement the missed care work in medicine and surgery, our findings cannot be generalized to other healthcare settings.

\section{CONCLUSION}

In today's modern healthcare world, the scope of nursing is expanding, with the risk of moving away from the provision of care that is fundamental to meet the patients' needs. Activities such as communication, helping patients during mealtimes, during mobilization and personal hygiene, maintaining their dignity, the provision of culturally congruent care, education in view of discharge to improve self-management, and emotional support, are the foundations for nursing care today and for the future.

If we continue to talk about missed nursing care only from the perspective of the professionals, without paying attention to the person as a whole, we run the risk of turning nursing care into a mere set of technical activities that are not centred on the real needs of the patient. Future research should aim at improving the essential component of nursing care, focusing namely on the patient, by adapting health services, hospital policies and procedures, so that they may 
support nurses in their mission to achieve the main goal of their profession: the psychophysical well-being of the patient.

\section{RELEVANCE TO CLINICAL PRACTICE}

This review enabled to highlight the importance of taking into account patients' perspectives throughout the healthcare process, because patients are the principal end users of the services that health organizations provide. The scientific literature of the last decade on the omission of nursing care, has demonstrated nurses' desire to improve the quality of care delivered, based on the awareness of the erosion that nursing care is undergoing, due to several reasons (e.g. the shortage of nurses). The intention to compare the results of studies that investigated the omission of activities from nurses' perceptions, with the results of studies that investigated patients' perceptions about unmet needs, is well-founded on the need to find a new gateway to the point of care in clinical practice. The actual healthcare landscape sees, on one side, patients increasingly competent related to their rights and health, on the other side, nurses increasingly conscious about their accountability for patient and healthcare outcomes. Knowing patients' point of view about their needs could help professionals to improve the quality and safety of care delivered, by responding more efficiently to patients' requests. Finally, although nurses often recognise in the same way as patients do, however patients may also identify unmet needs that are not perceived as such by nurses. Therefore, it could be useful to analyse available tools to measure missed care at the nurse level to align the data collection with the complexity of the patients' needs, as well outlined in the Fundamentals of Care Framework.

\section{Conflict of Interest statement}

The authors declare that they have no conflict of interest. 


\section{REFERENCES}

Abdel, M.A.S., Oweis, A.I., \& Hasna, F.S. (2012). Differences between patients' expectations and satisfaction with nursing care in a private hospital in Jordan. International Journal of Nursing Practice, 18(2), 140-146. DOI: 10.1111/j.1440-172X.2012.02008.x

Abu-El-Noor, M. K., \& Abu-El-Noor, N. I. (2014). Importance of Spiritual Care for Cardiac Patients Admitted to Coronary Care Units in the Gaza Strip: Patients' Perception. Journal of Holistic Nursing, 32(2), 104-115. DOI: 10.1177/0898010113503905

Aiken, L.H., Sloane, D.M., Bruyneel, L., Van den Heede, K., Griffiths, P., Busse, R., ... RN4CAST consortium. (2014). Nurse staffing and education and hospital mortality in nine European countries: a retrospective observational study. Lancet, 383(9931):1824-30. DOI: 10.1016/S0140-6736(13)62631-8.

Aiken, L. H., Sloane, D. M., Ball, J., Bruyneel, L., Rafferty, A. M., Griffiths, P. (2018). Patient satisfaction with hospital care and nurses in England: an observational study. BMJ Open, 11,8(1):e019189. DOI: 10.1136/bmjopen-2017-019189.

Alasad, J., Tabar, N. A., \& Aburuz, M. E. (2015). Patient satisfaction with nursing care: Measuring outcomes in an international setting. Journal of Nursing Administration, 45(11), 563568. DOI: 10.1097/NNA.0000000000000264. 
Alhusban, M. A., \& Abualrub, R. F. (2009). Patient satisfaction with nursing care in Jordan. Journal of Nursing Management, 17(6), 749-758. DOI: 10.1111/j.1365-2834.2008.00927.x

Arksey, H., \& O’Malley, L. (2005). Scoping studies: towards a methodological framework. International Journal of Social Research Methodology, 8(1), 19-32. DOI: $10.1080 / 1364557032000119616$

Ausserhofer, D., Zander, B., Busse, R., Schubert, M., De Geest, S., Rafferty, A.M., ... RN4CAST Consortium (2014). Prevalence, patterns and predictors of nursing care left undone in European hospitals: results from the multicountry cross-sectional RN4CAST study. BMJ Quality and Safety, 23, 126-35. DOI: 10.1136/bmjqs-2013-002318

Bachnick, S., Ausserhofer, D., Baernholdt, M., Simon, M., \& on behalf of the Match RN study group (2018). Patient-centered care, nurse work environment and implicit rationing of nursing care in Swiss acute care hospitals: A cross-sectional multi-center study. International Journal of Nursing Studies, 81, 98-106. DOI: 10.1016/j.ijnurstu.2017.11.007

Ball, J.E., Murrells, T., Rafferty, A.M., Morrow, E., Griffiths, P. (2014). 'Care left undone' during nursing shifts: associations with workload and perceived quality of care. BMJ Quality \& Safety, 23(2), 116-25. DOI: 10.1136/bmjqs-2012-001767. 
Ball, J., Day, T., Murrells, T., Dall'Ora, C., Rafferty, A. M., Griffiths, P., Maben, J. (2017). Cross-sectional examination of the association between shift length and hospital nurses job satisfaction and nurse reported quality measures. BMC Nursing, 25,16-26. DOI: 10.1186/s12912017-0221-7.

Ball, J. E., Bruyneel, L., Aiken, L. H., Sermeus, W., Sloane, D.M., Rafferty, A.M., ... RN4Cast Consortium. (2018). Post-operative mortality, missed care and nurse staffing in nine countries: A cross-sectional study. International Journal of Nursing Studies, 78,10-15. DOI: 10.1016/j.ijnurstu.2017.08.004.

Bishop, A. C., \& Macdonald, M. (2017). Patient involvement in patient safety: A qualitative study of nursing staff and patient perceptions. Journal of Patient Safety, 13(2), 82-87. DOI: 10.1097/PTS.0000000000000123

Bozimowski, G. (2012). Patient Perceptions of Pain Management Therapy: A Comparison of Real-Time Assessment of Patient Education and Satisfaction and Registered Nurse Perceptions. Pain Management Nursing, 13(4), 186-193. DOI: 10.1016/j.pmn.2010.04.004

Bruus, I., Varik, M., Aro, I., Kalam-Salminen, L., \& Routasalo, P. (2012). Patient-centeredness in long-term care of older patients - a structured interview. International Journal of Older People Nursing, 7(4), 264-271. DOI: 10.1111/j.1748-3743.2011.00301 
Bruyneel, L., Van Den Heede, K., Diya, L., \& Sermeus, W. (2009). Predictive validity of the international hospital outcomes study questionnaire: an RN4CAST Pilot Study. Journal of Nursing Scholarship, 41(2), 202-210. DOI: 10.1111/j.1547-5069.2009.01272.x

Cano-Plans, S., Lacueva-Pérez, L., Cabrera, E., \& Zabalegui, A. (2018). Knowledge expectations of orthopaedic patients. International Journal of Nursing Practice, 24(3), e12639. DOI: $10.1111 /$ ijn.12639

Cho E., Lee N. J., Kim E. Y., Kim S., Lee K., Park K. O., \& Sung Y. H. (2016). Nurse staffing level and overtime associated with patient safety, quality of care and care left undone in hospitals: A cross-sectional study. International Journal of Nursing Studies, 60, 263-271. DOI: 10.1016/j.jjnurstu.2016.05.009.

Crispin, V., Bugge, C., \& Stoddart, K. (2017). Sufficiency and relevance of information for inpatients in general ward settings: A qualitative exploration of information exchange between patients and nurses. International Journal of Nursing Studies, 75, 112-122. DOI: 10.1016/j.ijnurstu.2017.07.010

Critical Appraisal Skills Programme (2018). CASP Qualitative Checklist. Retrieved from: https://casp-uk.net/casp-tools-checklists/. Accessed: 19. 12. 2018. 
Dabney, B. W., \& Kalisch, B. J. (2015). Nurse Staffing Levels and Patient-Reported Missed Nursing Care. Journal of Nursing Care Quality, 30(4), 306-312. DOI: 10.1097/NCQ.0000000000000123

Doran, D. (2003). Nursing-sensitive outcome: state of the science. Sudbury, Massachuttes: Jones and Bartlett Publishers.

Dorigan, G. H., \& De Brito Guirardello, E. (2010). Patient satisfaction in a gastroenterology unit. ACTA Paulista de Enfermagem, 23(4), 500-505. DOI: 10.1590/S0103-21002010000400009

Ebrahimi, H., Torabizadeh, C., Mohammadi, E., \& Valizadeh, S. (2012). Patients' perception of dignity in Iranian healthcare settings: A qualitative content analysis. Journal of Medical Ethics, 38(12), 723-728. DOI: 10.1136/medethics-2011-100396

Feo, R., Kitson, A., \& Conroy T. (2018). How fundamental aspects of nursing care are defined in the literature: A scoping review. Journal of Clinical Nursing, 27(11-12), 2189-2229. DOI: 10.1111/jocn.14313.

Fuji, K. T., Abbott, A. A., \& Norris, J. F. (2013). Exploring Care Transitions From Patient, Caregiver, and Health-Care Provider Perspectives. Clinical Nursing Research, 22(3), 258-274. DOI: $10.1177 / 1054773812465084$ 
Gellerstedt, L., Medin, J., \& Karlsson, M. R. (2014). Patients' experiences of sleep in hospital: A qualitative interview study. Journal of Research in Nursing, 19(3), 176-188. DOI: $10.1177 / 1744987113490415$

Goh, M. L., Ang, E. N. K., Chan, Y.-H., He, H.-G., \& Vehviläinen-Julkunen, K. (2016). A descriptive quantitative study on multi-ethnic patient satisfaction with nursing care measured by the Revised Humane Caring Scale. Applied Nursing Research, 31, 126-131. DOI: 10.1016/j.apnr.2016.02.002

Griffiths, P., Ball, J. E., Drennan, J., James, L., Jones, J., Recio-Saucedo, A., \& Simon M. (2014). The association between patient safety outcomes and nurse/healthcare assistant skill mix and staffing levels and factors that may influence staffing requirements. NICE Evidence review, London: NICE.

Griffiths, P., Maruotti, A., Recio Saucedo, A., Redfern, O.C., Ball, J.E., Briggs, J., ... On behalf of Missed Care Study Group (2018). Nurse staffing, nursing assistants and hospital mortality: retrospective longitudinal cohort study. BMJ Quality and Safety Epub ahead of print: [please include 21.12.2018]. DOI: 10.1136/bmjqs-2018-008043

Griffiths, P., Recio-Saucedo, A., Dall'Ora, C., Briggs, J., Maruotti, A., Meredith, P., ... Missed Care Study Group (2018). The association between nurse staffing and omissions in nursing care: A systematic review. Journal of Advanced Nursing, 74(7), 1474-1487. DOI: 10.1111/jan.13564. 
Hayter, M. (2013). The UK Francis Report: the key messages for nursing. Journal of Advanced Nursing, 69(8), 1-3. doi: 10.1111/jan.12206.

Henderson, V. (1966). The Nature of Nursing: A Definition and its Implications for Practice, Research, and Education. New York: Macmillan Publishing.

Institute of Medicine (US) - Committee on Quality of Health Care in America (2001). Crossing the Quality Chasm: A New Health System for the 21st Century. Washington (DC): National Academies Press (US).

Johansson Stark, Å., Ingadottir, B., Salanterä, S., Sigurdardottir, A., Valkeapää, K., BachrachLindström, M., \& Unosson, M. (2014). Fulfilment of knowledge expectations and emotional state among people undergoing hip replacement: A multi-national survey. International Journal of Nursing Studies, 51(11), 1491-1499. DOI: 10.1016/j.ijnurstu.2014.03.006

Jones, T.L., Hamilton, P., \& Murry, N. (2015). Unfinished nursing care, missed care, and implicitly rationed care: State of the science review. International Journal of Nursing Studies, 52, 1121-37. DOI: 10.1016/j.ijnurstu.2015.02.012.

Kalisch, B.J., \& Williams, R.A. (2009). Development and psychometric testing of a tool to measure missed nursing care. Journal of Nursing Administration, 39(5), 211-9. DOI: 10.1097/NNA.0b013e3181a23cf5. 
Kalisch, B.J., \& Xie, B. (2014). Errors of Omission: Missed Nursing Care. Western Journal of Nursing Research, 36(7), 875-90. DOI: 10.1177/0193945914531859.

Kalisch, B.J., Landstrom, G.L., \& Hinshaw, A.S. (2009). Missed nursing care: a concept analysis. Journal of Advanced Nursing, 65(7), 1509-17. DOI: 10.1111/j.1365-2648.2009.05027.

Kalisch, B.J., McLaughlin, M., \& Dabney, B.W. (2012). Patient Perceptions of Missed Nursing Care. The Joint Commission Journal on Quality and Patient Safety, 38(4), 161-167.

Kalisch, B.J., Xie, B., \& Dabney, B.W. (2014) Patient-Reported Missed Nursing Care Correlated with Adverse Events American. Journal of Medical Quality, 29(5), 415-422. DOI: $10.1177 / 1062860613501715$.

Kelley, K., Clark, B., Brown, V., \& Sitzia, J. (2003). Good practice in the conduct and reporting of survey research. International Journal for quality in Health Care, 15(3), 261-266.

Kitson, A. (2016). Why Do We Need to Study the Fundamentals of Care? Nursing Leadership, 29(1), 10-16. DOI:10.12927/cjnl.2016.24641

Kitson, A.L., \& Muntlin Athlin, Å. (2013). Development and preliminary testing of a framework to evaluate patients' experiences of the fundamentals of care: a secondary analysis of three stroke survivor narratives. Nursing Research and Practice: 572437. DOI: 10.1155/2013/572437. 
Kitson, A.L., Muntlin Athlin, Å, Elliott, J., \& Cant, M.L. (2013). What's my line? A narrative review and synthesis of the literature on Registered Nurses' communication behaviours between shifts. Journal of Advanced Nursing, 70(6):1228-42. doi:10.1111/jan.12321.

Koskenniemi, J., Leino-Kilpi, H., Puukka, P., Stolt, M., \& Suhonen, R. (2018). Being respected by nurses: Measuring older patients' perceptions. International Journal of Older People Nursing, 13(3), e12197. DOI: 10.1111/opn.12197

Laird, E. A., McCance, T., McCormack, B., \& Gribben, B. (2014). Patients' experiences of inhospital care when nursing staff were engaged in a practice development programme to promote person-centredness: A narrative analysis study. International Journal of Nursing Studies, 52(9), 1454-1462. DOI: 10.1016/j.ijnurstu.2015.05.002

Lake, E.T., Germack, H.D., Viscardi, M.K. (2016). Missed nursing care is linked to patient satisfaction: a cross-sectional study of US hospitals. BMJ Quality \& Safety, 25(7), 535-43. doi: 10.1136/bmjqs-2015-003961.

Land, L., \& Suhonen, R. (2009). Orthopaedic and trauma patients' perceptions of individualized care. International Nursing Review, 56(1), 131-137. DOI: 10.1111/j.1466-7657.2008.00688.x

Lei, Z., Qiongjing, Y., Qiuli, W., Sabrina, K., Xiaojing, L., \& Changli, W. (2009). Sleep quality and sleep disturbing factors of inpatients in a Chinese general hospital. Journal of Clinical Nursing, 18(17), 2521-2529. DOI: 10.1111/j.1365-2702.2009.02846.x 
Leininger, M. M. (1995). Transcultural nursing: Concepts, theories, research \& practices. New York: McGraw Hill.

Lin, Y.-P., Tsai, Y.-F., \& Chen, H.-F. (2011). Dignity in care in the hospital setting from patients' perspectives in Taiwan: A descriptive qualitative study. Journal of Clinical Nursing, 20(5-6), 794-801. DOI: 10.1111/j.1365-2702.2010.03499.x

Mahjoub, R., \& Rutledge, D. N. (2011). Perceptions of informed consent for care practices: Hospitalized patients and nurses. Applied Nursing Research, 24(4), 276-280. DOI: 10.1016/j.apnr.2009.12.001

Malmgren, M., Törnvall, E., \& Jansson, I. (2014). Patients with hip fracture: Experiences of participation in care. International Journal of Orthopaedic and Trauma Nursing, 18(3), 143150. DOI: $10.1016 /$ j.ijotn.2013.08.017

Maslow, A.H. (1943) A Theory of Human Motivation. Psychological Review, 50, 370-396. DOI: $10.1037 / \mathrm{h} 0054346$.

Mbuzi, V., Fulbrook, P., \& Jessup, M. (2017). Indigenous cardiac patients' and relatives' experiences of hospitalisation: A narrative inquiry. Journal of Clinical Nursing, 26(23-24), 5052-5064. DOI: $10.1111 /$ jocn.14005 
Montin, L., Johansson, K., Kettunen, J., Katajisto, J., \& Leino-Kilpi, H. (2010). Total joint arthroplasty patients' perception of received knowledge of care. Orthopaedic Nursing, 29(4), 246-253 DOI: 10.1097/NOR.0b013e3181e51868

Mukasa, J. P., Glass, N., \& Mnatzaganian, G. (2015). Ethnicity and patient satisfaction with tuberculosis care: A cross-sectional study. Nursing and Health Sciences, 17(3), 395-401. DOI: $10.1111 /$ nhs. 12202

Needleman, J. (2016). The Economic Case for Fundamental Nursing Care. Nursing Leadership (Toronto, Ontario). 2016;29(1):26-36.

Oetker-Black, S., \& Petrochuk, M. (2012). A descriptive analysis of nursing satisfaction: firsttime versus non-first-time medical-surgical patients. Health Marketing Quarterly, 29(4), 303310. DOI: $10.1080 / 07359683.2012 .732851$

Oflaz, F., \& Vural, H. (2010). The evaluation of nurses and nursing activities through the perceptions of inpatients. International Nursing Review, 57(2), 232-239. DOI: 10.1111/j.14667657.2009.00772.x

Palese, A., Tomietto, M., Suhonen, R., Efstathiou, G., Tsangari, H., Merkouris, A., ... Papastavrou, E. (2011). Surgical patient satisfaction as an outcome of nurses' caring behaviors: A descriptive and correlational study in six European countries. Journal of Nursing Scholarship, 43(4), 341-350. DOI: 10.1111/j.1547-5069.2011.01413.x 
Papastavrou, E., Andreou, P., \& Efstathiou, G. (2014) Rationing of nursing care and nursepatient outcomes: a systematic review of quantitative studies. The International Journal of Health Planning and Management, 29, 3-25. DOI: 10.1002/hpm.2160.

Papastavrou, E., Efstathiou, G., Tsangari, H., Suhonen, R., Leino-Kilpi, H., Patiraki, E., ... Merkouris, A. (2012). Patients' and nurses' perceptions of respect and human presence through caring behaviours: A comparative study. Nursing Ethics, 19(3), 369-379. DOI: $10.1177 / 0969733011436027$

Portillo, M. C., \& Cowley, S. (2011). Working the way up in neurological rehabilitation: The holistic approach of nursing care. Journal of Clinical Nursing, 20(11-12), 1731-1743. DOI: 10.1111/j.1365-2702.2010.03379.x

Radecki, B., Reynolds, S., \& Kara, A. (2018). Inpatient fall prevention from the patient's perspective: A qualitative study. Applied Nursing Research, 43, 114-119. DOI: 10.1016/j.apnr.2018.08.001

Rasmussen, T. S., \& Delmar, C. (2014). Dignity as an empirical lifeworld construction-In the field of surgery in Denmark. International Journal of Qualitative Studies on Health and WellBeing, 9(1). DOI: 10.3402/qhw.v9.24849 
Ringdal, M., Chaboyer, W., Ulin, K., Bucknall, T., \& Oxelmark, L. (2017). Patient preferences for participation in patient care and safety activities in hospitals. BMC Nursing, 16(1). DOI: $10.1186 / \mathrm{s} 12912-017-0266-7$

Royal College of Nursing (2017). Safe and Effective Staffing: Nursing Against the Odds. London: Royal College of Nursing.

Sasso, L., Bagnasco, A., Aleo, G., Catania, G., Dasso, N., Zanini, M.P., \& Watson, R. (2017). Incorporating nursing complexity in reimbursement coding system: the potential impact on missed care. BMJ Quality \& Safety, 26(11):929-932. doi: 10.1136/bmjqs-2017-006622.

Sasso, L., Bagnasco, A., Zanini, M., Catania, G., Aleo, G., Santullo, A., ... Sermeus, W. (2017). The general results of the RN4CAST survey in Italy. Journal of Advanced Nursing, 73(9), 20282030. DOI: $10.1111 /$ jan.13066.

Sasso, L., Bagnasco, A., Catania, G., Zanini, M., Aleo, G., Watson, R. (2019). Push and pull factors of nurses' intention to leave. Journal of Nursing Management, 27(5), 946-954. DOI:10.1111/jonm.12745.

Sayin, Y., \& Aksoy, G. (2012). The Nurse's Role in Providing Information to Surgical Patients and Family Members in Turkey: A Descriptive Study. AORN Journal, 95(6), 772-787. DOI: 10.1016/j.aorn.2011.06.012. 
Schubert, M., Glass, T.R., Clarke, S.P., Schaffert-Witvliet, B., \& De Geest, S. (2007). Validation of the basel extent of rationing of nursing care instrument. Nursing Research, 56, 416-24.

Sermeus, W., Aiken, L.H., Van Den Heede, K., Rafferty, A.M., Griffiths, P., Moreno-Casbas, M.T., ... \& RN4CAST Consortium (2011). Nurse forecasting in Europe (RN4CAST): Rationale, design, and methodology. BMC Nursing, 10,6. DOI: 10.1186/1472-6955-10-6.

Shoqirat, N. (2014). "Sleepless nights and sore operation site": Patients' experiences of nursing pain management after surgery in Jordan. Pain Management Nursing, 15(3), 609-618. DOI: 10.1016/j.pmn.2013.05.002

Squires, A., Aiken, L.H., van den Heede, K., Sermeus, W., Bruyneel, L., Lindqvist, R., Schoonhoven, L., ... \& Matthews, A. (2012). 'A systematic survey instrument translation process for multi-country, comparative health workforce studies', International Journal of Nursing Studies, 50(2), 264-73. doi: 10.1016/j.ijnurstu.2012.02.015.

Suhonen, R., Berg, A., Idvall, E., Kalafati, M., Katajisto, J., Land, L., ... Leino-Kilpi, H. (2009). European orthopaedic and trauma patients' perceptions of nursing care: A comparative study. Journal of Clinical Nursing, 18(20), 2818-2829. DOI: 10.1111/j.1365-2702.2009.02833.x

Suhonen, R., Papastavrou, E., Efstathiou, G., Tsangari, H., Jarosova, D., Leino-Kilpi, H., ... Merkouris, A. (2012). Patient satisfaction as an outcome of individualised nursing care. 
Scandinavian Journal of Caring Sciences, 26(2), 372-380. DOI: 10.1111/j.14716712.2011.00943.x

Suhonen, R., Stolt, M., Habermann, M., Hjaltadottir, I., Vryonides, S., Tonnessen, S., ... RANCARE Consortium (2018). Ethical elements in priority setting in nursing care: A scoping review. International Journal of Nursing Studies, 17(88), 25-42. DOI: 10.1016/j.ijnurstu.2018.08.006

Twibell, R. S., Siela, D., Sproat, T., \& Coers, G. (2015). Perceptions related to falls and fall prevention among hospitalized adults. American Journal of Critical Care, 24(5), e78-e85. DOI: 10.4037/ajcc2015375

Van Bokhorst-De Van Der Schueren, M. A. E., Roosemalen, M. M., Weijs, P. J. M., \& Langius, J. A. E. (2012). High waste contributes to low food intake in hospitalized patients. Nutrition in Clinical Practice, 27(2), 274-280. DOI: 10.1177/0884533611433602

World Health Organization, \& Global Health Workforce Alliance (2013). A universal truth: no health without a workforce. Switzerland (Geneva): World Health Organization. Retrieved from: https://www.who.int/patientsafety/patients_for_patient/programme/en/ [Accessed: 10.12.2018]

Yiu, H. Y., Chien, W.-T., Lui, M. H.-L., \& Qin, B. (2011). Information needs of Chinese surgical patients on discharge: a comparison of patients' and nurses' perceptions. Journal of Advanced Nursing, 67(5), 1041-1052. DOI: 10.1111/j.1365-2648.2010.05528.x 
Zander, B., Dobler, L., Bäumler, M., Busse, R. (2014). [Nursing tasks left undone in German acute care hospitals - results from the international study RN4Cast]. Gesundheitswesen, 76(11),727-34. DOI: 10.1055/s-0033-1364016.

Zhang, J. P., Yao, S. Q., Ye, M., Huang, H. S., He, G. P., \& Leng, X. H. (2009). A study on the subjective well-being and its influential factors in chronically ill inpatients in Changsha, China. Applied Nursing Research, 22(4), 250-257. DOI: 10.1016/j.apnr.2008.02.005 


\section{Figure 1 PRISMA 2009 Flow Diagram}

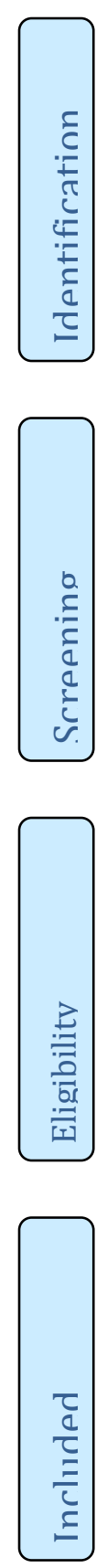

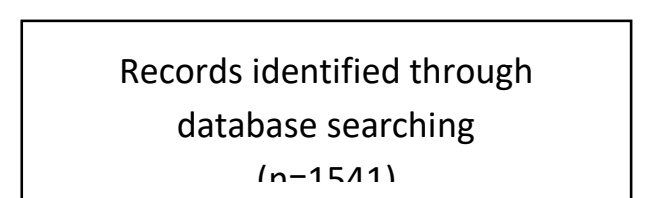

In=15/1)
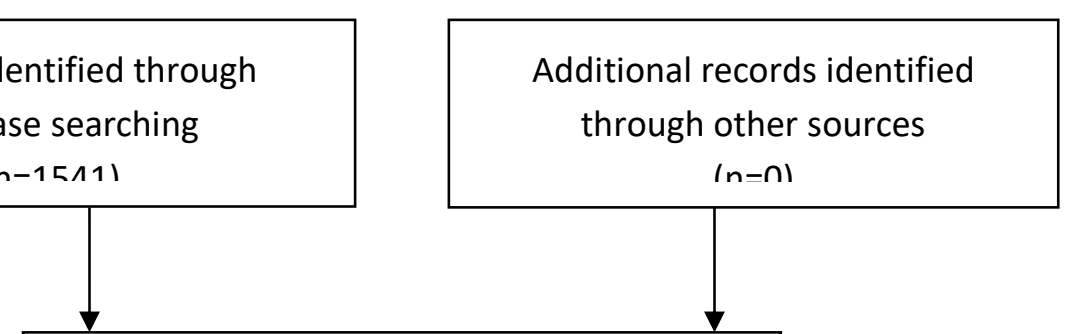

Records after duplicates removed

$(n=1428)$
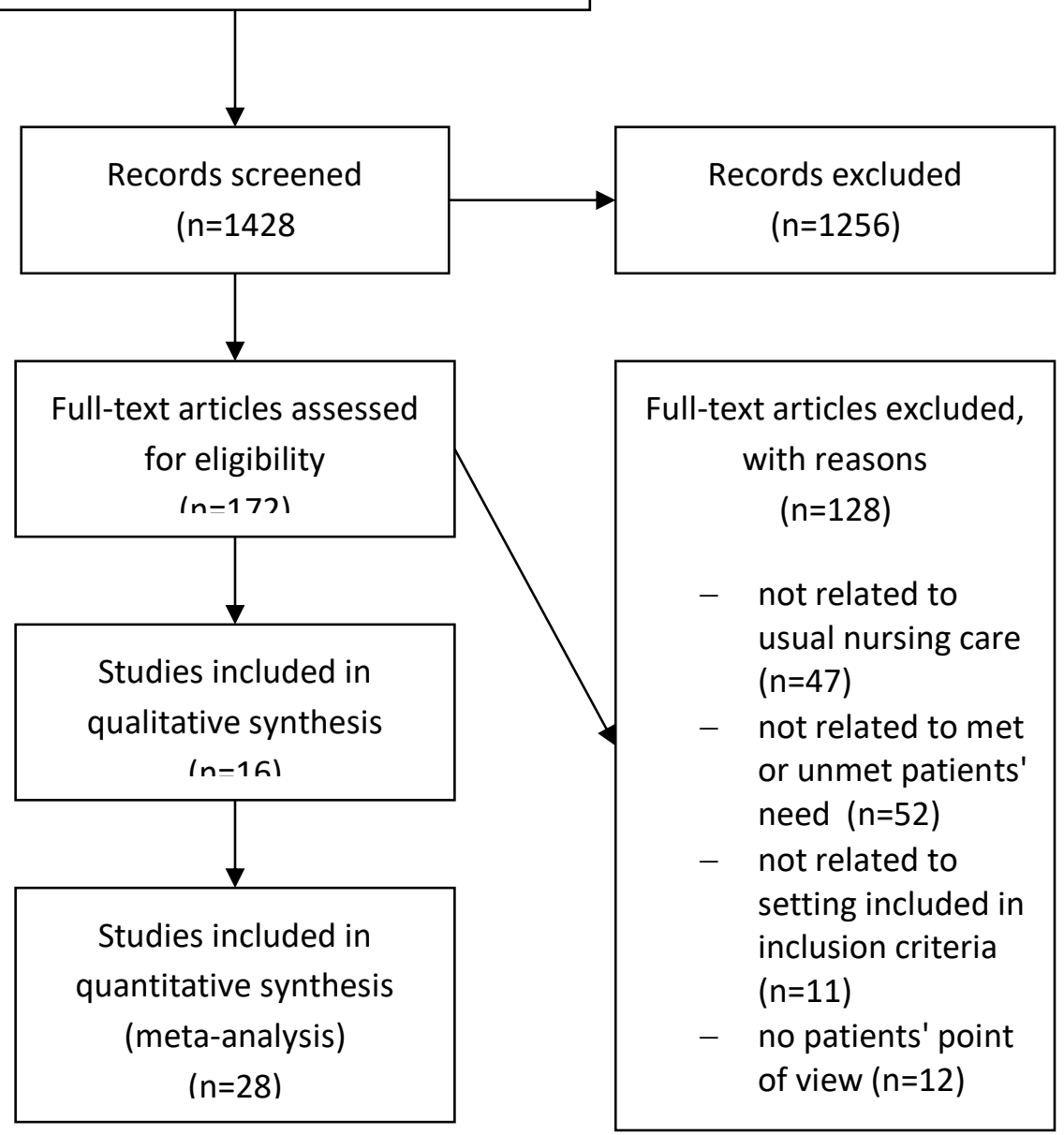


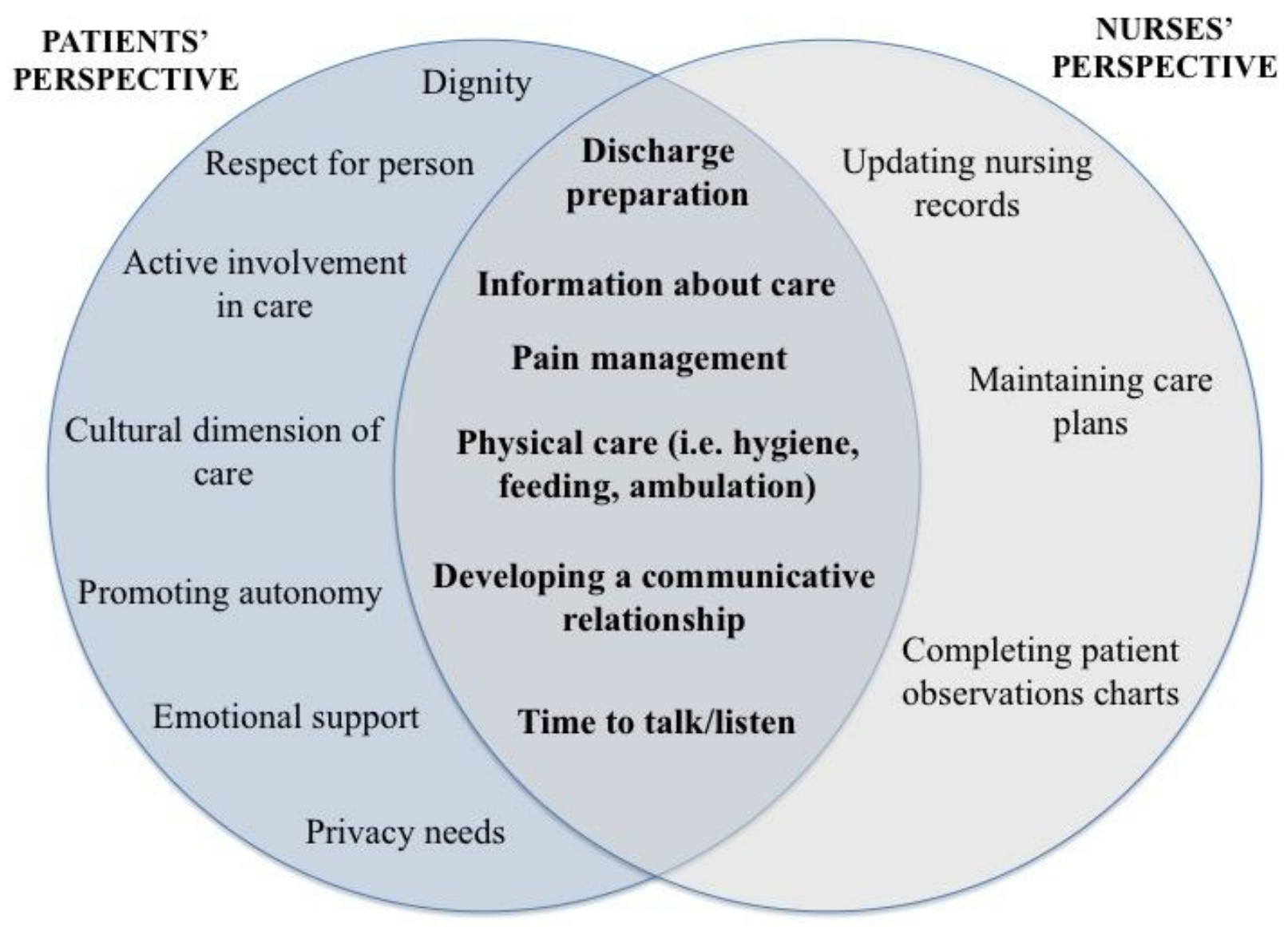

